Theme: Oxygen Steelmaking

\title{
LIGHT METER: A RELIABLE WAY TO PREDICT BOF END POINT CARBON*
}

\author{
Henrique Silva Furtado ${ }^{1}$ \\ Tathagata Bhattacharya ${ }^{2}$ \\ Daniel Pandolfi ${ }^{3}$
}

\begin{abstract}
Light meter is an ArcelorMittal innovation that uses off-gas light intensity to predict bath carbon, which is akin to the visual cues that the operators employ to finish a blow. Light meter enables the prediction of end point that is independent of any operator's judgment. The results have been in general consistent with the actual carbon content at BOF end point at Tubarão. Besides some outliers that occurred due to strange intensity curves, the system implemented in Tubarão BOF 3 reported a carbon prediction error statistic that is very similar to dissolved oxygen-carbon end point statistics. Preliminary trials using light meter without any filter improved carbon hit ratio significantly.
\end{abstract}

Keywords: BOF process; End point control; Carbon prediction; Off gas light intensity.

1 Metallurgical Engineer, PhD, Specialist in Steelmaking, ArcelorMittal Tubarão, Serra, ES, Brazil.

2 Process Modeler, PhD, Research Engineer, Steelmaking \& Refractory ArcelorMittal Global R\&D, Chicago, IN, USA.

3 Mechanical Technologist, Automation post graduate, Specialist in electric and electronic reliability, Steelmaking Department, ArcelorMittal Tubarão, Serra, ES, Brazil.

* Technical contribution to the $45^{\text {th }}$ Steelmaking Seminar, May $25^{\text {th }}-28^{\text {th }}, 2014$, Porto Alegre, RS, Brazil. 


\section{INTRODUCTION}

Basic oxygen furnace (BOF) is a high-productivity process to refine steel from a mixture of hot metal and scrap. Like other high temperature reactors, however, it lacks a reliable sensor that might control its process evolution in a better way and therefore, its capacity in attending the aimed condition at the end of the process.

Any BOF process (only top-blown, combined top oxygen blown with bottom inert gas injection or combined top and bottom oxygen blown or only bottom oxygen blown) uses a so called static model to design the amount of charge, fluxes, coolants and oxygen to be used to achieve an aimed end of process condition for steel (e.g., temperature; $\mathrm{C}$ and $\mathrm{P}$ contents). These hybrid models are based on thermo chemical calculation with some statistics that aims at incorporating actual past data to predict the end point.

Although, static models are useful for setting up of heats, they have serious limitations when it comes to predicting the endpoint due to their inability to handle process dynamics that reflect the change in the process over time (the "trajectory" information of a heat). Also, uncertainty in the initial conditions (such as hot metal temperature, composition, etc.) and process drifts due to lance operation, refractory wear, change in bath height, etc. contribute to heat-to-heat variation that cannot be accounted for in the static model. Therefore, sole reliance on static charge models results in either under-blowing or over-blowing of BOF heats, which has a significant impact on productivity, and costs due to lower iron yield, extra coolants and aluminum consumption, for example.

In order to improve the end point hit ratio (the attainment of aimed end point condition), sublance system was developed. At present, installed in many moderns steelmaking shops, they usually perform in-blow measurements close to the end of blowing using a special sensor to measure liquid metal temperature and predict carbon content from solidification algorithm. Using this information, a predictive model is run and sets up a correction to be done in oxygen and coolant additions, in order to achieve the aimed end point conditions. Despite a significant improvement this system has provided, steelmakers still have to deal with over or under blown heats at the end of a blow, which is in general due to the level of accuracy of temperature measurements and predicted carbon as well as the model limitation to predict the evolution of the remaining blowing period.

BOF off-gas analysis is also used with relative success and sometimes may be a solution to substitute a more expensive sublance system. However, depending on the requested differences between actual end point condition and the aimed one, offgas based system may also not be enough to achieve high end point hit ratio.

A combination of different systems may improve BOF end point achievements further; however, this will mean more investment costs. Following in this direction, but with a minimal cost (ArcelorMittal Tubarão already has a sublance system), it was decided to test a patented device [1] that measures light intensity from the mouth of a converter during blow, named carbon light meter (CLM). Light intensity at the end of blow has been used by operators to predict carbon for long time. However, it is very dependent on their skills. As light intensity actually carries decarburization information from bath, a proper instrumentation and a reliable algorithm may improve this prediction for all operators independent of their skills.

* Technical contribution to the $45^{\text {th }}$ Steelmaking Seminar, May $25^{\text {th }}-28^{\text {th }}, 2014$, Porto Alegre, RS, Brazil. 


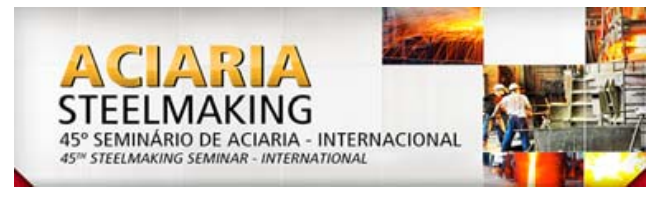

At present, CLM has been applied to open hood BOF process [2]. The present work aims at presenting the first set of industrial trials using CLM in ArcelorMittal Tubarao converter 3, which uses a closed hood system for gas recovery.

\section{MATERIAL AND METHODS}

Light meter sensor was installed in front of BOF 3 control pulpit, around $25 \mathrm{~m}$ far from the converter mouth (Figure 1). Its signal was collected every 10 milliseconds through a cable connecting CLM to a PLC where carbon prediction is done.

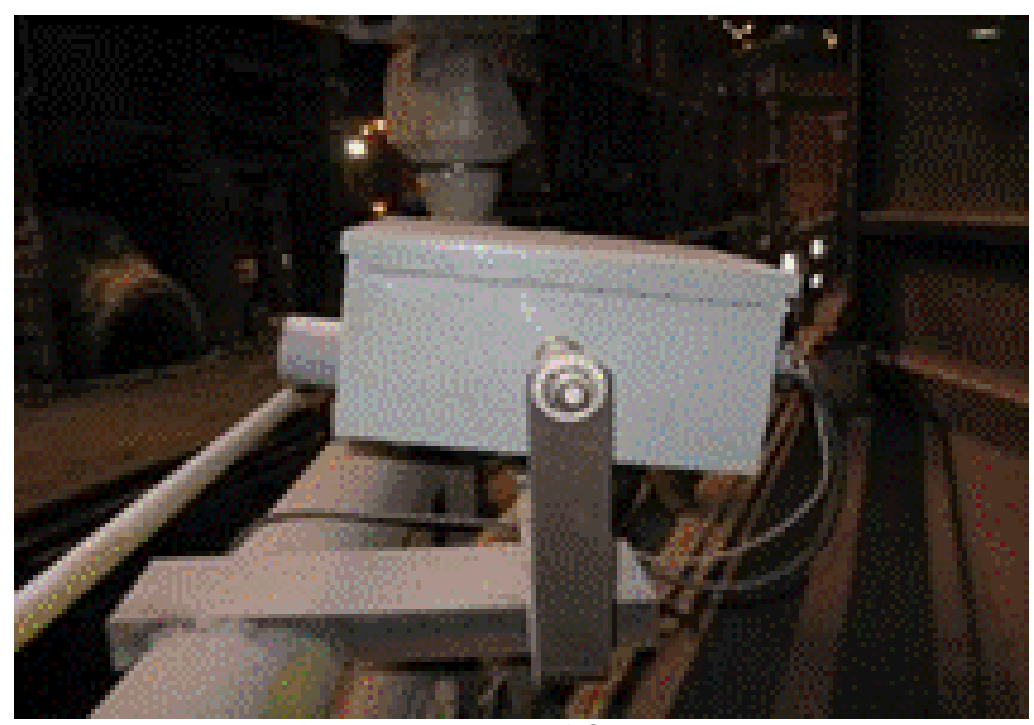

Figure 1. A view of CLM sensor

After in-blow sublance measurements, the converter's hood opens up automatically making it possible to expose the flame at the mouth of the vessel. This moment triggers light meter data collection and calculations. The results are dynamically displayed to operators during this last part of oxygen blow through a simple temporary screen implemented on their computer. However, the decision to stop blow were still kept in operator' hands. A typical light meter curve can be seen in Figure 2.

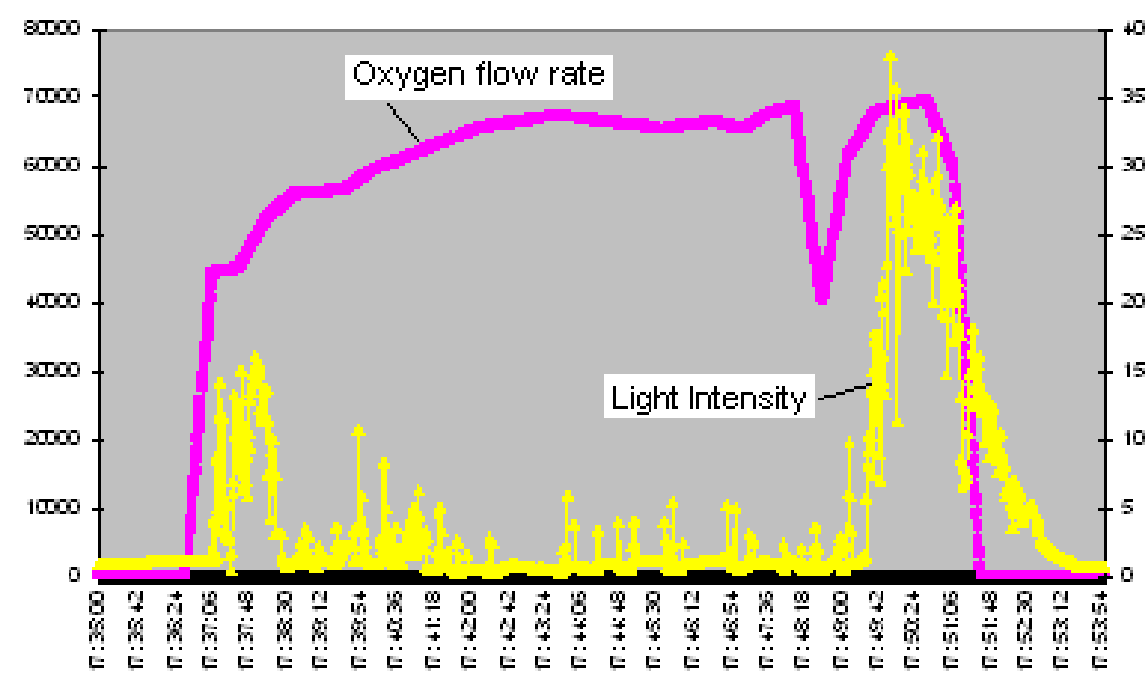

Figure 2. A typical light meter signal of one heat.

* Technical contribution to the $45^{\text {th }}$ Steelmaking Seminar, May $25^{\text {th }}-28^{\text {th }}, 2014$, Porto Alegre, RS, Brazil. 


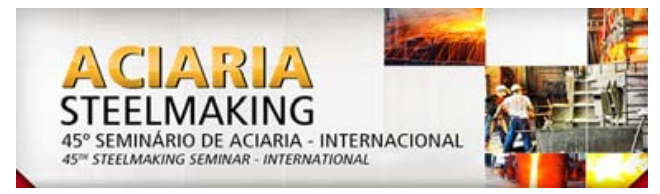

During the period of CLM tests no special operational procedure was implemented. Blowing parameter was determined according to our operational standards.

Some BOF end point measurements (temperature, sample analysis and oxygen activity) were performed according to our standards. Then, CLM carbon prediction was compared with a reference carbon analysis from Tubarão spectrometer. As a comparison with CLM prediction, carbon prediction from oxygen activity (Celox) and deviation from spectrometer was also analyzed.

\section{RESULTS AND ANALYSIS}

In order to verify the results of CLM predicted carbon (CLM C), one determines the difference (error) between the reported values with sample results from spectrometer analysis ( standard Tubarão BOF end point analysis), as shown in Figure 3.

From Figure 3, one can observe that the mean of the difference between CLM C and spectrometer is very close to zero. This is an indication of good accuracy. However, there is a scatter in the data that seems to be biased to the right hand side (signifying over prediction). Also, an outlier can be observed on the left side of Figure 3.

The data scattering observed in figure 3 has two main sources: one came from the model that predicts carbon from light intensity, which is not $100 \%$ perfect; and the second one is due to variability in light intensity itself. This last one can be due to: oxygen flow rate after in-blow sublance measurements; hood pressure variation; movable hood opening delay; totally closed dog house; materials addition or any other event that might change the light intensity during the last stage of blow.

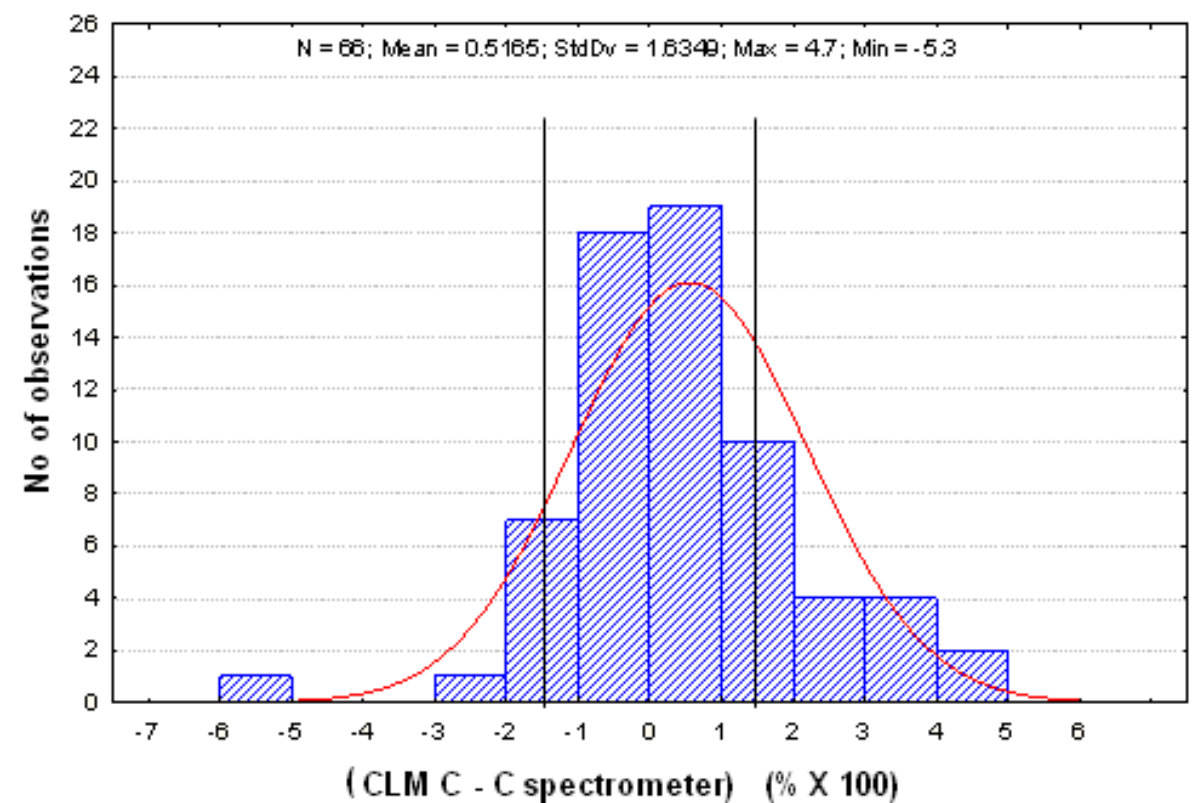

Figure 3. Typical error statistics of carbon prediction from light meter.

One typical light meter curve that reproduced very high differences between CLM prediction and spectrometer measurement is shown in figure 4. In that specific heat, the actual end point C was $0.17 \%$ while CLM predicted C was only $0.045 \%$. This is an extreme example where the operator stopped the blow at a high $\mathrm{C}$ level, where light intensity was still high, indicating that some carbon was still burning. At this situation CLM C prediction is not reliable, because CLM model needs a dropping

* Technical contribution to the $45^{\text {th }}$ Steelmaking Seminar, May $25^{\text {th }}-28^{\text {th }}, 2014$, Porto Alegre, RS, Brazil. 
light intensity curve. Therefore, some kind of filters must be developed in order to reduce the probability of having outliers in $\mathrm{C}$ prediction.

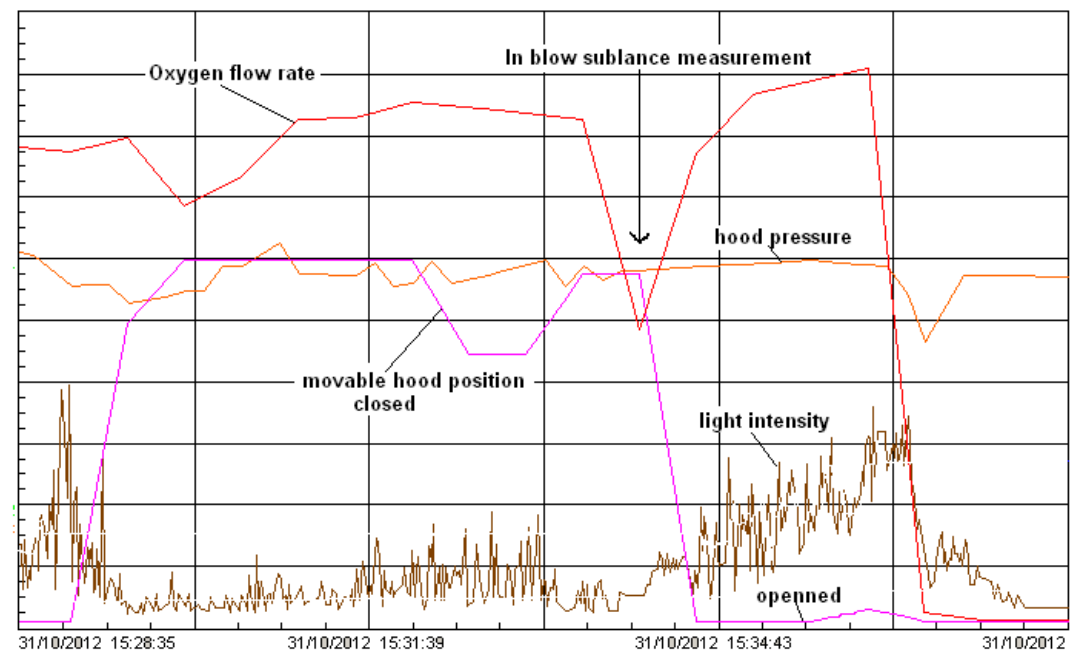

Figure 4. An example of a situation where an anomalous prediction was made from the CLM light intensity curve.

Table I summarize some statistics on carbon prediction errors. As can be seen, the lower the CLM C, the more accurate is the prediction. However, the chance to achieve BOF end point with a very low $C$ content increases at low CLM C prediction range. In cases where ultra low carbon is the focus of production or direct tapping is the case to maximize production, this may not be a problem. However, lower carbon is generally followed by high oxygen activity, which means lower iron yield and higher Al consumption. Therefore, there remains a considerable room for improvement in the predictive model system.

Table 1. Some statistics on carbon hit ratio

\begin{tabular}{|c|c|c|c|}
\hline \multirow{2}{*}{ CLM C prediction } & \multicolumn{3}{|c|}{ PROBABILITIES (\%) } \\
\cline { 2 - 4 } & $\begin{array}{c}\text { To hit aimed end point } \\
\text { C range }\end{array}$ & $\begin{array}{c}\text { To have actual C }> \\
\text { aimed C }+0.015 \%\end{array}$ & $\begin{array}{c}\text { To have actual C }< \\
\text { aimed C }-0.010 \%\end{array}$ \\
\hline$>=3$ and $<4$ & 78 & 0.3 & 22 \\
\hline$>=4$ and $<5$ & 72 & 13 & 15 \\
\hline$>=5$ and $<6$ & 65 & 32 & 3 \\
\hline$>=6$ & 18 & 72 & 11 \\
\hline
\end{tabular}

Despite the above discussion on CLM C prediction issues, for the same set of data of Figure 3 (trial data), these errors were statistically very similar to those determined by Celox measurements (compare Figure 3 and 5). However, Celox carbon prediction is still more precise especially for situations where CLM one turned out to be an outlier, as for example, the heat illustrated in figure 4 for which the actual carbon content was very high (Celox reported $0.14 \%$ C).

* Technical contribution to the $45^{\text {th }}$ Steelmaking Seminar, May $25^{\text {th }}-28^{\text {th }}, 2014$, Porto Alegre, RS, Brazil. 

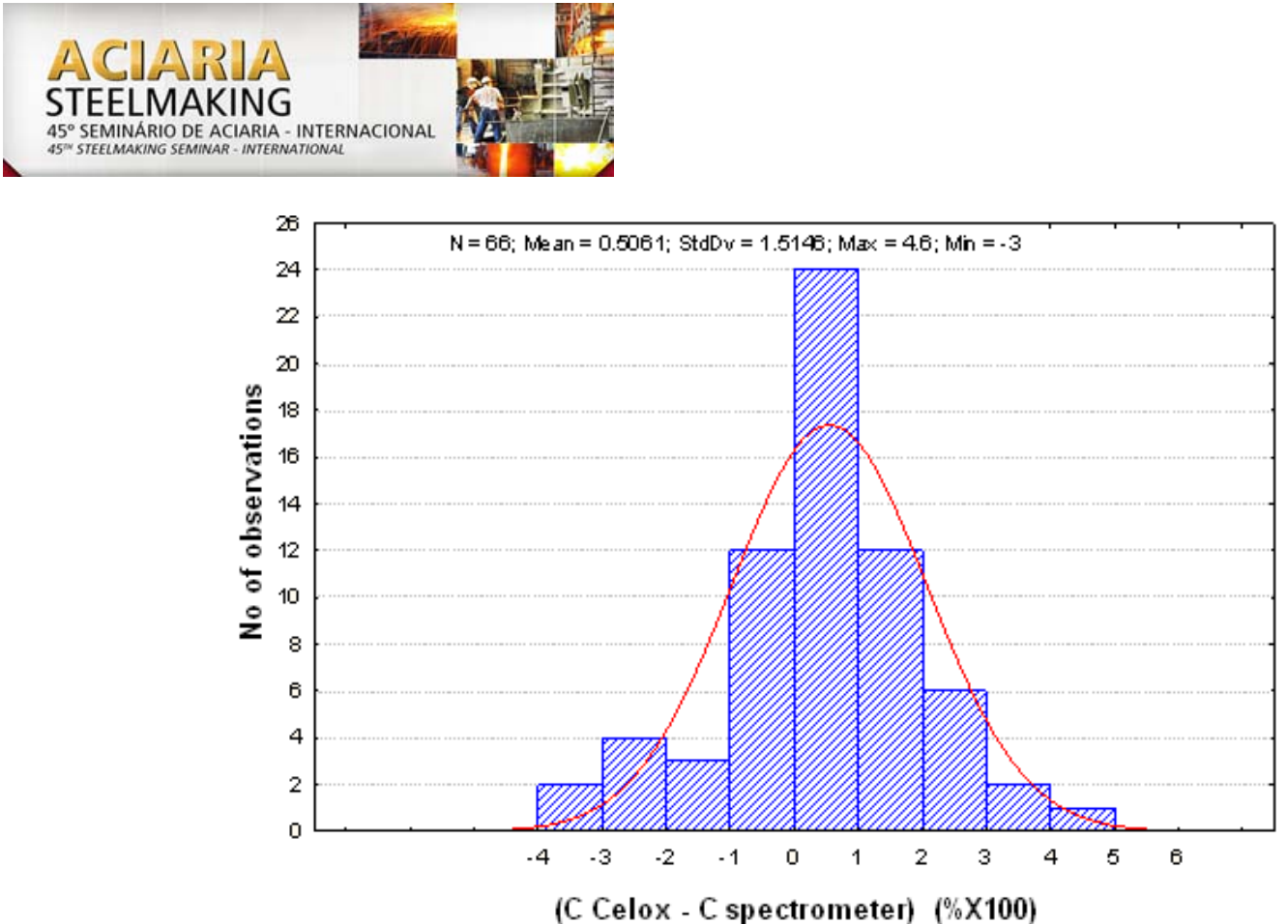

Figure 5. Typical error statistics of carbon predicted by Celox.

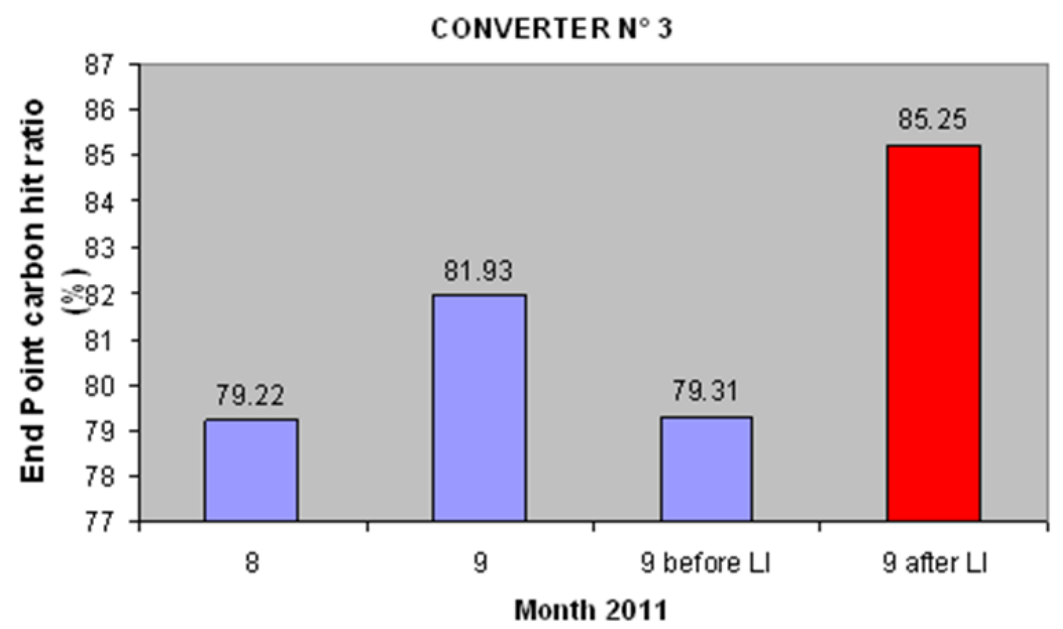

Figure 6. Carbon hit ratio before and after blowing end as guided by carbon light meter

\section{CONCLUSIONS}

ArcelorMittal patented system that uses light intensity to predict the end of BOF process carbon was successfully tested at ArcelorMital Tubarão site converter 3.

The accuracy of light meter in predicting end point carbon during trials was high when compared with spectrometer results.

Light meter prediction can be erroneous sometimes due to many factors such as model quality; oxygen flow rate after in blow sublance measurements; hood pressure variation; movable hood opening delay; totally closed dog house; materials addition or any other event that might affect the light intensity during the last stage of blow.

In order to deliver good carbon estimations, light meter also needs a dropping light intensity curve during the period after in blow sublance measurements.

Carbon hit ratio increases for low carbon heats. However, it also increases the chance to actually have very low carbon content at the end of blow, which may not be a problem for ultra-low carbon production or if the goal is direct tapping, but may reduce iron yield and increase Al consumption.

* Technical contribution to the $45^{\text {th }}$ Steelmaking Seminar, May $25^{\text {th }}-28^{\text {th }}, 2014$, Porto Alegre, RS, Brazil. 
During the trial period, error statistics from light meter predicted carbon were very close to measurements based on oxygen activity; however, oxygen activity still produces more precise end point carbon estimation, manly in situations of unexpected light intensity curves or higher carbon levels.

During a period when end point was advised by light meter, carbon hit ratio increased significantly.

\section{REFERENCES}

1 Sharan A. Method and apparatus to determine and control the carbon content of steel in a BOF vessel. United States Patent, n. 5, p.603-746, Feb. 18, 1997.

2 Bhattacharya T, Shah P, Chukwulebe B. Development and application of an endpoint control (EPC) model for an open-hood converter. In: AISTech Conference Proceedings; 2013. Warrendale : AIST; 2013. p.364-303.

* Technical contribution to the $45^{\text {th }}$ Steelmaking Seminar, May $25^{\text {th }}-28^{\text {th }}, 2014$, Porto Alegre, RS, Brazil. 